\title{
Relation between the Size of Lamellar Droplets in Onion Phases and Their Effective Surface Tension
}

\author{
E. van der Linden and W. T. Hogervorst \\ Unilever Research Laboratories, Vlaardingen, The Netherlands \\ H. N. W. Lekkerkerker* \\ Van't Hoff Laboratory for Physical and Colloid Chemistry, Utrecht University, \\ P.O. Box 80.051, 3508 TB Utrecht, The Netherlands \\ Received October 30, 1995. In Final Form: February 12, 1996 ${ }^{\otimes}$
}

\begin{abstract}
A cl osed packed system ("onion phase") of so-called lamellar dropl ets, consisting of concentric surfactant bilayers which are separated from one another by water, may be obtained by subjecting a continuous lamellar phase to shear. We propose a model for the size of these lamellar droplets under shear as a function of their effective surface tension. This model fits the available experimental data quantitatively and explains the dependence of the size on surfactant volume fraction.
\end{abstract}

\section{Introduction}

Surfactants in solution can form supramolecular aggregates of various constitutions, e.g. micelles, vesicles, and sheets of bilayers. ${ }^{1}$ The bilayer sheets may form stacks of parallel bilayers, thus forming a lamellar or $L_{\alpha}$ phase. $^{2}$ Under certain conditions, a dispersion may be formed, ${ }^{3,4}$ of which one of the two phases consists of socalled lamellar droplets, 5 (or "onions" 7,8), where the bilayers are stacked like the layers in an onion ${ }^{5,9}$ and are separated from one another by water. This configuration has led to the obvious classification of these dispersions as "onion phases". The dispersing phase may either be an ordinary isotropic phase or a lamellar phase. In the remaining part of this paper, we will only consider the latter.

Recently a method of preparing such onion phases with a narrow droplet size distribution has been reported by Diat et al. 7,8 The narrow size distribution was obtained by subjecting a continuous lamellar phase to a constant shear rate. Although the effect of shear on a lamellar phase has received attention al ready for over a decade, ${ }^{10-12}$ the actual processes leading to droplet formation itself are still under debate. ${ }^{8}$ Diat et al..$^{7,8}$ have observed a relation between the size, $D$, of lamellar droplets in an onion phase and the applied shear rate, $\dot{\gamma}$, during formation: $\mathrm{D} \sim 1 / \sqrt{ } \dot{\gamma}$. They present a model, based on a bal ance between the viscous stress and the elastic stress stored in the onion, which explains this experimental observation. However, as they havealsoremarked, ${ }^{8}$ their model does not explain correctly the dependence of size

\footnotetext{
* Corresponding author.

${ }^{\otimes}$ Abstract published in AdvanceACS Abstracts, April 15, 1996.

(1) See e.g. the Introduction to Physics of amphiphiles: Micelles, Vesicles and Microemulsions; Degiorgio, V., Corti, M., Eds.; Elsevier Science Publishers B.V.: Amsterdam, 1985.

(2) Ekwall, P. In Advances in Liquid Crystals; Brown, G. M., Ed.; Academic Press: New York, 1975.

(3) Kunieda, H.; Shinoda, K.J . Phys. Chem. 1978, 82, 1711.

(4) Dubois, M.; Zemb, Th. Langmuir 1991, 7, 1352.

(5) Pas, J. C. van de. Tenside, Surfactants, Deterg. 1991, 28, 3.

(6) Pas, J. C. van de. A study of the physical properties of lamellar liquid-crystalline dispersions. Ph.D. Thesis, University of Groningen, 1993.

(7) Diat, O.; Roux, D. J . Phys. II 1993, 3, 9.

(8) Diat, O.; Roux, D.; Nallet, F. J. Phys. II 1993, 3, 1427.

(9) Gray, G. W.; Winsor, P. W. Liq. Cryst. Plast. Cryst. 1974, 1, 223.

(10) Oswald, P.; Ben-Abraham, S. I. J . Phys. (Paris) 1982, 43, 1193.

(11) Safinya, C. R.; Sirota, E. B.; Bruinsma, R. F.; eppesen, C.; Plano,

R. J .; Wenzel, L. J . Science 1993, 261, 588.

(12) Bruinsma, R.; Rabin, Y. Phys. Rev. A 1992, 45, 994.
}

on surfactant volume fraction, $\phi$, at a given shear rate. Their model predicts a $\sqrt{ } \phi$ dependence, while their experiments yield a $\phi^{2}$ dependence.

Here we propose an alternative model for the dependence of onion size on shear rate and surfactant volume fraction. It is again based on a balancebetween theviscous stress and an elastic stress. The latter now contains bilayer interaction contributions. To validate our model the dependence of the viscous stress on the shear rate was measured and the relation between the onion size and the viscous stress was determined. This again leads in good approximation to $D \sim 1 / \sqrt{ } \dot{\gamma}$, whereas the bilayer interactions ultimately account for the $\phi^{2}$ dependence.

\section{Model}

We consider a close packed system (so-called "onion phase") of "monodisperse"lamellar droplets ("onions")..$^{5-9}$ As found by Diat et al.,7,8 such a system is obtained by subjecting a continuous lamellar phase (a "real" one-phase system ${ }^{6}$ ) to a shear flow as present in a Couette cell, consisting of two concentriccylinders, of which onecylinder is given an angular velocity with respect to the other (a practically constant shear rate is obtained in the small gap between thecylinders). Wenotethat the onion phase will not show phase separation by virtue of sufficiently high repulsive bilayer interactions (insideonedroplet and between droplets), which also accounted for the stability of the original continuous lamellar phase, and as such is very different from an onion phase where the dispersing phase is an ordinary continuous phase.

The process of actual droplet formation out of a continuous lamellar phaseis still subject to discussion. 8,11,12 However, the existence of droplets and characteristic droplet sizes has been establi ished. ${ }^{3-8}$ These droplet sizes are usually, i.e. in the case of normal emulsions, determined by the maximum allowed deformation before breakup and a balance between the shear stress on the droplet by the surrounding medium versus the surface stress, which is related to the presence of the (curved and deformed) droplet surface and its surface tension. ${ }^{13-15}$

However, onion phases arefundamentally different from ordinary emulsions in at least tworespects. First, lamellar

(13) Taylor, G. I. Proc. R. Soc. London, Ser. A 1934, 146, 501.

(14) Silberberg, A.; Kuhn, W. Nature 1952, 170, 450.

(15) Silberberg, A.; Kuhn, W. J . Polym. Sci. 1954, 13, 21. 
droplets exhibit an internal structure, which is absent in emulsion droplets. Second, the droplets can only be distinguished from their surroundings because of thefact that the curvature of their outer bilayer is different from theaveragecurvature of their surroundings. For therest, thephases insideand outsidethedroplets are equivalent. In that respect, an onion phase may be regarded as a one-phase system with a large number of defects in the form of onions. ${ }^{16}$ This is further exemplified by the fact that the here described onion phases are transparent, indicating that the refractive indexes of the onion and its surroundings are not different from one another.

The difference in curvature of the droplet surface with respect toits surroundings implies, during shear, a surface stress, equal to the Laplace pressure, $4 \sigma_{\text {eff }} / D$, where $D$ and $\sigma_{\text {eff }}$ denotethe diameter and (effective) surfacetension of the droplet, respectively. ${ }^{16,17}$ This Laplace pressure must equal the local shear stress. This local stress stems from the stress caused by the moving boundaries, which penetrates into the onion phase over a characteristic distance, say $L$, where $L \gg D .{ }^{18}$ If the gap width, $G$, of the Couette device is smaller than $L$, penetration of this stress is ensured throughout the whole sample. In our experiments, $1 \mu \mathrm{m}<\mathrm{D}<10 \mu \mathrm{m}, \mathrm{L} \approx(1-100) \mathrm{mm},{ }^{18}$ and $\mathrm{G}=1 \mathrm{~mm}$, satisfying $\mathrm{G}<\mathrm{L}$. Hence, thelocal shear stress will equal theexperimentally determined shear stress, as measured during preparation.

We conclude that, in the model described above, the surface stress, $4 \sigma_{\text {eff }} / D$, dueto the curvature of thesurface, created at a certain shear rate $\dot{\gamma}$, balances theshear stress, $\eta \dot{\gamma}$, where $\eta$ is the experimentally determined viscosity of the onion phase at shear rate $\dot{\gamma}$. According to this model, one then finds for the onion diameter

$$
\mathrm{D}=4 \sigma_{\mathrm{eff}} / \eta \dot{\gamma}
$$

Wenotethat Taylor ${ }^{13}$ and Sil berbergand Kuhn ${ }^{14,15}$ have also predicted this scaling behavior, for ordinary emulsions, but the expressions all differ from each other in the prefactor. In our model, the phases at theinner and outer side are completely equivalent and the viscosity which thedroplet experiences will bethetotal medium viscosity.

To deduce from eq 1 the dependence of droplet size on shear rate $\dot{\gamma}$ and surfactant volume fraction $\phi$, we need to establish on the onehand therelation between viscosity and $\dot{\gamma}$ and $\phi$ and on the other hand the relation between surface tension and $\phi$.

\section{Viscous Effects}

We prepared different onion phases, using different shear rates, containing a constant concentration of $30 \%$ AOT (sodium salt of di(2-ethyl hexyl) sul fosuccinate, used as received from Sigma, approximately $99 \%$ pure) in a brine solution (10 g/L NaCl p.a., used as received from Merck, in doubly distilled water).

Theshear cell used was a Haake Rotoviscometer RV20, an apparatus consisting of two concentric cylinders, of which onecylinder (theinner cylinder) is given an angular velocity with respect to the other (outer cylinder). The shear rateprofilefor Couetteflow in our case, with respect to a gap size, G, of $1 \mathrm{~mm}$, is practically constant (within $5 \%$ deviation). During our experiments the Haake Rotoviscometer was mounted with an interchangeable head M10 and a spindle (inner cylinder) MV1, which gave us an adjustableshear range of $0.08-880 \mathrm{~s}^{-1}$. A $60 \mathrm{~g}$ sample

(16) Boltenhagen, P.; Kleman, M.; Lavrentovich, O. D. J . Phys. II 1994, 4, 1439.

(17) Van der Linden, E.; Dröge, J . H. M. Physica A 1993, 193, 439.

(18) De Gennes, P. G. The Physics of Liquid Crystals; Oxford University Press: Oxford, 1974.

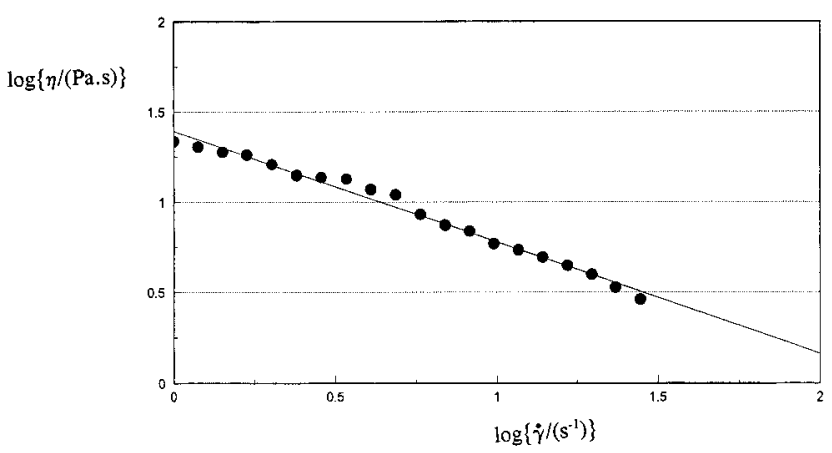

Figure 1. Viscosity, $\eta$, versus shear rate, $\dot{\gamma}$, of an onion phase containing droplets $7.5 \mu \mathrm{m}$ in diameter, prepared at a shear rate of $30 \mathrm{~s}^{-1}$.

containing AOT in brinesolution $(10 \mathrm{~g} / \mathrm{L} \mathrm{NaCl})$ is prepared in a $500 \mathrm{~mL}$ conical flask, mounted with a reflux condenser. Theflask is placed on a heating plate and heated to around $230{ }^{\circ} \mathrm{C}$. The sample is heated and stirred for $15 \mathrm{~min}$ to dissolve the AOT in the brine solution. The temperature of the AOT sample reaches a maximum of $110{ }^{\circ} \mathrm{C}$. After these $15 \mathrm{~min}$ the stirring is stopped, but the heating is continued for $10 \mathrm{~min}$. At this point the heating is stopped and the sample is cautiously stirred to mix the solution coming from the condenser with the AOT sample. This preparation results in a viscous continuous lamellar AOT sample (checked with light microscopy) with a very smal I amount of lamellar droplets. This mixture is directly poured into the beaker of the Haake apparatus and, in or der to have it homogeneousl y distributed, sheared at 10 $\mathrm{s}^{-1}$ for $10 \mathrm{~min}$, at a temperature of $95^{\circ} \mathrm{C}$. Subsequently, the temperature of the mixture was lowered cautiously down to $25^{\circ} \mathrm{C}$, without shear. It was checked at this stage whether the sample was continuous lamellar, in order to ensure equivalent initial conditions before applying shear to the samples. Subsequently the AOT sample was sheared at the desired shear rate until the applied shear stress remained constant (usually after about $20 \mathrm{~min}$ ). Thus, a monodisperse sample consisting of lamellar droplets with a characteristic size was obtained. The characteristicsize of thelamellar droplets was determined using light microscopy.

We measured the viscosity of each sample (after the preparation) as a function of shear rate. Weonly measured at shear rates lower than the one which was used to preparethesample. Thus wemadesurethat thedroplets would retain their size during the measurement, as was also checked afterward experimentally. In Figure 1 the dependence of viscosity on shear rateis shown for an onion phase which was prepared at $30 \mathrm{~s}^{-1}$, containing droplets with a diameter of about $7.5 \mu \mathrm{m}$. Analogously, one may determine a viscosity versus shear rate profile for each onion phase, containing droplets with a certain diameter. Writing

$$
\log \{\eta /(\mathrm{Pa} \cdot \mathrm{s})\}=\mathrm{k}_{2}+\mathrm{k}_{1} \log \left\{\dot{\gamma} /\left(\mathrm{s}^{-1}\right)\right\}
$$

one may determine $k_{1}$ and $k_{2}$ for various onion phases having different droplet diameters. In Figures 2 and 3 , $\mathrm{k}_{1}$ and $\mathrm{k}_{2}$ are shown as a function of droplet diameter, respectively. F or $k_{1}$ wefind a value of -0.55 , independent of droplet diameter. Alsofor $\mathrm{k}_{2}$ no systematic dependence on droplet diameter is found, although its value of 0.9 only has an accuracy of $30 \%$. Using eq 2 , we conclude from our experiments

$$
\eta /(\mathrm{Pa} \cdot \mathrm{s})=8\left\{\dot{\gamma} /\left(\mathrm{s}^{-1}\right)\right\}^{-0.55}
$$

which does not contain any dependence on $\mathrm{D}$. We note 


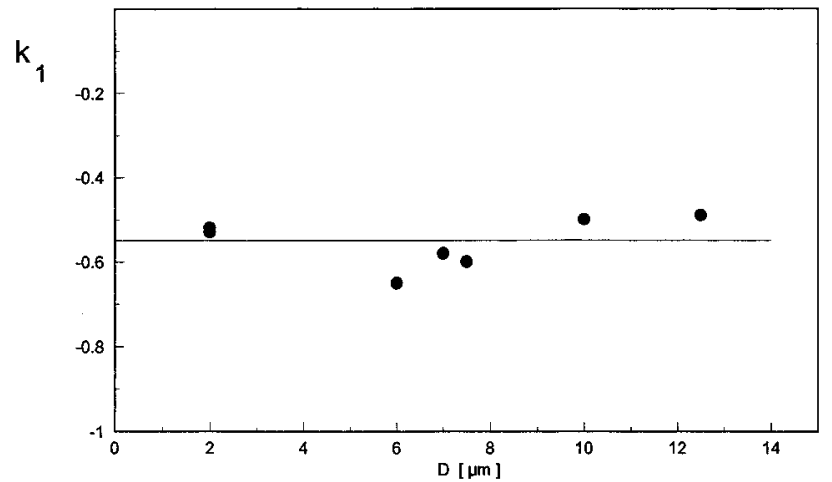

Figure 2. Dependence of $k_{1}$ on droplet diameter, D.

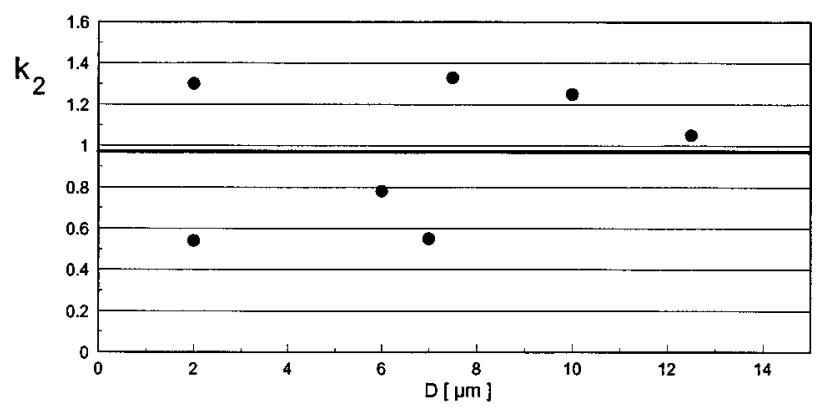

Figure 3. Dependence of $k_{2}$ on droplet diameter, D.

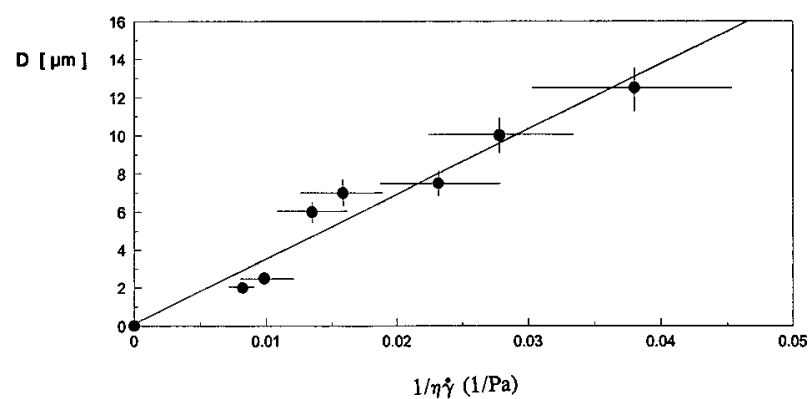

Figure4. Droplet diameter, D, versus $1 / \eta \dot{\gamma}$. Theslopeisrelated to the effective surface tension of a lamellar droplet.

that a similar expression has also been reported by Bohlin ${ }^{19}$ and by Bohlin and Fontel ${ }^{20}$ for lamellar phases. They found to leading order in the surfactant volume fraction $\phi, \eta /(\mathrm{Pa} \cdot \mathrm{s})=5.4\left\{\dot{\gamma} / \mathrm{s}^{-1}\right\}^{-1 / 2}$ for a $30 \% \mathrm{CTAB}$ - hexan-1-olwater system.

By means of eq 3, one calculates the shear stress corresponding totheshear ratewhich was used to prepare the droplets. In Figure 4, D is plotted against this shear stress. We find a linear relationship, as predicted by our model, between onion size and the inverse shear stress (for the $30 \%$ AOT system)

$$
\mathrm{D} /(\mathrm{m})=0.36 \times 10^{-3} /\left\{\eta \dot{\gamma} /\left(\mathrm{N} / \mathrm{m}^{2}\right)\right\}
$$

\section{Surface Tension}

Recently, one of us ${ }^{17}$ obtained an expression for the effective surface tension $\sigma_{\text {eff }}$ of a lamellar droplet

$$
\sigma_{\text {eff }}=\{\mid(\mid+1)(\mid+2)(\mid-1) K B\}^{1 / 2} /(\mid+2)(\mid-1)
$$

wherel denotes the deformation mode, $\mathrm{K}=\kappa / \mathrm{d}$ with $\mathrm{d}$ the smectic repeating distance between the bilayers in the droplet and $\kappa$ the bilayer rigidity, and $\mathrm{B}=\mathrm{d}\left(\partial^{2} \mathrm{E}_{\mathrm{a}} / \partial \mathrm{D}^{2}\right)$

(19) Bohlin, L. J . Colloid Interface Sci. 1979, 69, 194.

(20) Bohlin, L.; Fontell, K. J . Colloid Interface Sci. 1978, 67, 272. with $E_{a}$ the bilayer interaction per unit area. ${ }^{21-24}$ For I $\rightarrow \infty$ one obtains

$$
\sigma_{\text {eff }}=(\mathrm{KB})^{1 / 2}
$$

which is the expression for the effective surface tension for a planar stack. ${ }^{18,25}$ Note that eqs 5 and 6 differ from eqs 4.7 and 4.8 in ref 17 , respectively, by a factor $1 / 2$, which had been erroneously added in that reference. For the case of AOT bilayers in high salt, but in fact for any system where the lamellar phase is stabilized by Helfrich interactions, one has ${ }^{24,26-27}$

$$
B=9 \pi^{2}(k T)^{2} d / 64 \kappa d_{w}{ }^{4}
$$

where $\mathrm{k}$ is Boltmann's constant, $\mathrm{T}$ is the temperature, and $\mathrm{d}=\delta+\mathrm{d}_{\mathrm{w}}$, with $\delta$ and $\mathrm{d}_{\mathrm{w}}$ denoting the thicknesses of the bilayer and the water layer, respectively. Consequently one finds ${ }^{17}$

$$
\sigma_{\text {eff }} /\left(\mathrm{N} \cdot \mathrm{m}^{-1}\right)=0.48 /\left(\mathrm{d}_{\mathrm{w}} / \AA\right)^{2}
$$

One may write $d_{w}$ in terms of $\phi$ as

$$
\mathrm{d}_{\mathrm{w}}=\delta(1-\phi) / \phi
$$

Substituting eq 9 into eq 8 yields

$$
\sigma_{\text {eff }} /\left(\mathrm{N} \cdot \mathrm{m}^{-1}\right)=0.48 \phi^{2} /\left\{(1-\phi)^{2}(\delta / \AA)^{2}\right\}
$$

To leading order in $\phi$ this becomes

$$
\sigma_{\text {eff }} /\left(\mathrm{N} \cdot \mathrm{m}^{-1}\right)=0.48 \phi^{2} /(\delta / \AA)^{2}
$$

\section{Discussion}

Firstly, we address the shear rate dependence Combining our model, eq 1 , with eq 3 and rounding off the resulting exponent -0.45 to $-1 / 2$ implies a scaling of $D$ with the shear rate, as reported experimentally by Diat et al. 7,8

$$
\mathrm{D} /(\mathrm{m}) \sim 1 / \sqrt{ }\left\{\dot{\gamma} /\left(\mathrm{s}^{-1}\right)\right\}
$$

Secondly, we address the vol ume fraction dependence. Substituting eq 11 into eq 1, combined with eq 3, and using the leading order term in $\phi$ of the viscosity, one obtains

$$
\left.\mathrm{D} /(\mathrm{m})=0.24 \phi^{2} /\{(\delta / \AA))^{2}\right\} \sqrt{ }\left\{\dot{\gamma} / \mathrm{s}^{-1}\right\}
$$

where the coefficient 0.24 is valid for AOT. Assuming that this coefficient does not vary greatly from system to system and using a reasonable value of $\delta=40 \AA$ for the "bilayer" thickness in the system studied by Diat et al.., 21 (based on the length of two SDS $\mathrm{C}_{12}$-chains of $22 \AA$ and a waterlayer thickness of $18 \AA^{21}$ ), we find

$$
\mathrm{D} \sqrt{ } \dot{\gamma} /\left(\mathrm{m} \cdot \mathrm{s}^{-1 / 2}\right)=\left(1.5 \times 10^{-4}\right) \phi^{2}
$$

Taking e.g. $\phi=0.1$ gives $\mathrm{D} \sqrt{ } \dot{\gamma}=1.5 \times 10^{-6} \mathrm{~m} \cdot \mathrm{s}^{-1 / 2}=1.5$

(21) Safinya, C. R.; Roux, D.; Smith, G. S.; Sinha, S. K.; Dimon, P.; Clark, N. A.; Bellocq, A. M. Phys. Rev. Lett. 1986, 57, 2718.

(22) Leibler, S.; Lypowski, R. Phys. Rev. B 1987, 35, 7004.

(23) Roux, D.; Safinya, C. R. J . Phys. (Paris) 1988, 49, 307.

(24) Nallet, F. Langmuir 1991, 7, 1861.

(25) Durand, M. G. C. R. Acad. Sci. Paris B 1972, 275, 629.

(26) Helfrich, W. Z. Naturforsch. 1978, A33, 305.

(27) Nallet, F.; Roux, D.; Prost, J . Phys. Rev. Lett. 1989, 62, 276. 
$\mu \mathrm{m} \cdot \mathrm{s}^{-1 / 2}$, which is in satisfactory agreement with a value of about $1 \mu \mathrm{m} \cdot \mathrm{s}^{-1 / 2}$, as estimated from Figure 30 of ref 8 .

Finally we note that the experimentally valid eq 4 in combination with eq 1 allows us to deduce a value of 0.1 $\mathrm{mN} / \mathrm{m}$ for the surface tension of the AOT system studied here. Substituting a reasonable value for $\delta$ in the theoretically obtained eq $11(\delta=30 \AA)$ leads to a number of the same order of magnitude.

Note Added in Proof. While this paper was being

(28) Bergenholtz, J .; Wagner, N. J . Langmuir 1996, 12, 3122. reviewed, wel earned that Bergenholtz and Wagner ${ }^{28}$ also found their data in agreement with a scaling of the onion size with the inverse of the shear stress.

Acknowledgment. We thank J ohn van de Pas for discussions and for pointing out ref 19 , and we also acknowledge Edgar Blokhuis, Dick Bedeaux, J orrit Mellema, and Marijn Warmoeskerken for discussions. 